\title{
Emphysematous cystitis associated with voiding dysfunction from herpes zoster
}

\author{
Takeshi Kondo MD
}

Cite as: CMAJ 2019 June 3;191:E614. doi: 10.1503/cmaj.181542

A n 85-year-old woman presented to the clinic with a 3-day history of fever. She had been diagnosed with sacral herpes zoster 2 weeks earlier and was prescribed a 7-day course of valacyclovir. One week after the rash appeared, she developed symptoms (poor urine flow and sensation of incomplete emptying of the bladder) of underactive bladder. On physical examination, the patient had painful erythemas with scabs in the S1-S3 dermatomes (Supplementary Figure 1, Appendix 1, available at www.cmaj.ca/lookup/suppl/doi:10.1503/cmaj.181542/-/DC1) and suprapubic tenderness. Urinalysis showed an elevated leukocyte count. Computed tomography of the pelvis showed submucosal emphysematous changes in the bladder wall (Figure 1), consistent with emphysematous cystitis. Escherichia coli was identified in urine and blood cultures. The patient's fever resolved after treatment with antibiotics and bladder catheterization, and her symptoms of underactive bladder resolved spontaneously after 3 months of self-catheterization.

Herpes zoster is a result of reactivation of endogenous infection of varicella-zoster virus in the latent form within the sensory ganglia. In particular, S2 and S3 herpes zoster can cause voiding dysfunction after appearance of the skin rash, although it rarely affects the sacral dermatomes. Herpes zoster can affect bladder function by causing cystitis, neuritis or myelitis. ${ }^{1,2}$ Cystitis involves direct invasion of the bladder wall by the varicella-zoster virus, resulting in dysuria and increased urinary frequency or retention. ${ }^{1,2}$ In neuritis, the varicella-zoster virus in the unilateral sacral dorsal root ganglion spreads into the bilateral sacral motor nerves, interrupting the bilateral detrusor reflex, which manifests as an underactive bladder, as in this patient. ${ }^{1,2}$ In myelitis, suprasacral spinal cord inflammation causes a spastic bladder. ${ }^{1,2}$

Emphysematous cystitis is an uncommon form of urinary tract infection that may be complicated by sepsis and is characterized by gas formation within the bladder wall and lumen. Older women and patients with diabetes mellitus, obstructive uropathy and neurogenic bladder are predisposed to emphysematous cystitis. ${ }^{3}$ In this patient, it is likely that her older age and underactive bladder from sacral herpes zoster-related neuritis contributed to the development of emphysematous cystitis.

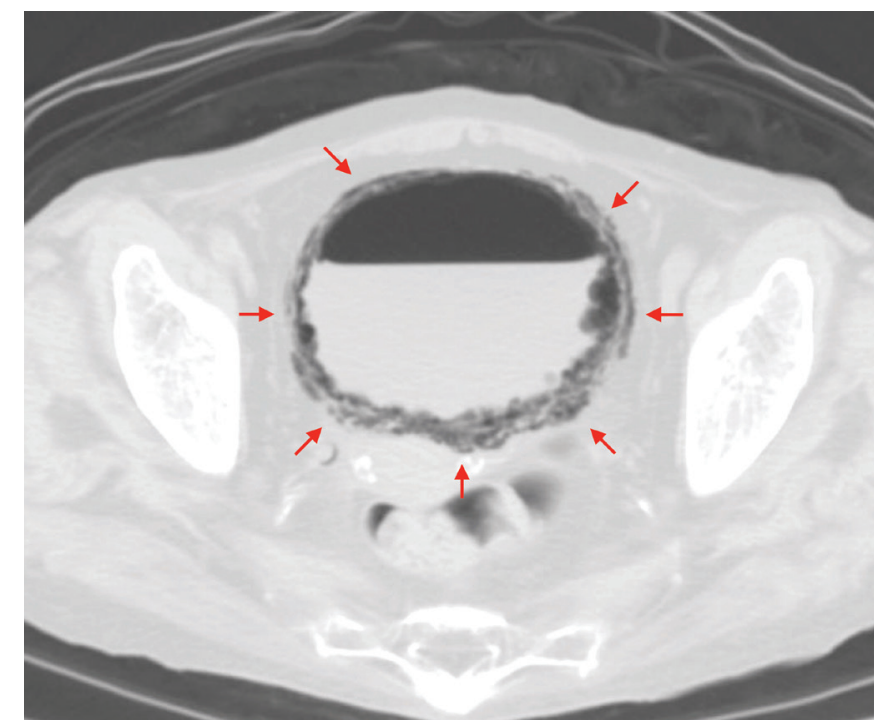

Figure 1: Computed tomography of the pelvis showing submucosal emphysema (arrows) in the bladder wall of an 85-year-old woman with fever, sacral herpes zoster and underactive bladder.

\section{References}

1. Marques SA, Hortense J. Herpes zoster-associated acute urinary retention in immunocompetent patient. An Bras Dermatol 2014;89:985-7.

2. Jakubovicz D, Solway E, Orth P. Herpes zoster: unusual cause of acute urinary retention and constipation. Can Fam Physician 2013;59:e146-7.

3. Amano M, Shimizu T. Emphysematous cystitis: a review of the literature. Intern Med 2014;53:79-82.

\section{Competing interests: None declared.}

This article has been peer reviewed.

The author has obtained patient consent.

Affiliation: Department of General Medicine, Chiba University Hospital, Chiba City, Chiba, Japan

Correspondence to: Takeshi Kondo, reds_liverpool_2005@yahoo.co.jp 